\title{
Multi-Graph Multi-Label Learning Based on Entropy
}

\author{
Zixuan Zhu and Yuhai Zhao * \\ College of Computer Science and Engineering, Northeastern University, Shenyang 110819, China; \\ 20141929@stu.neu.edu.cn \\ * Correspondence: zhaoyuhai@mail.neu.edu.cn
}

Received: 25 March 2018; Accepted: 30 March 2018; Published: 2 April 2018

\begin{abstract}
Recently, Multi-Graph Learning was proposed as the extension of Multi-Instance Learning and has achieved some successes. However, to the best of our knowledge, currently, there is no study working on Multi-Graph Multi-Label Learning, where each object is represented as a bag containing a number of graphs and each bag is marked with multiple class labels. It is an interesting problem existing in many applications, such as image classification, medicinal analysis and so on. In this paper, we propose an innovate algorithm to address the problem. Firstly, it uses more precise structures, multiple Graphs, instead of Instances to represent an image so that the classification accuracy could be improved. Then, it uses multiple labels as the output to eliminate the semantic ambiguity of the image. Furthermore, it calculates the entropy to mine the informative subgraphs instead of just mining the frequent subgraphs, which enables selecting the more accurate features for the classification. Lastly, since the current algorithms cannot directly deal with graph-structures, we degenerate the Multi-Graph Multi-Label Learning into the Multi-Instance Multi-Label Learning in order to solve it by MIML-ELM (Improving Multi-Instance Multi-Label Learning by Extreme Learning Machine). The performance study shows that our algorithm outperforms the competitors in terms of both effectiveness and efficiency.
\end{abstract}

Keywords: multi-graph multi-label; entropy; informative subgraphs; extreme learning machine

\section{Introduction}

Due to the advance of smart phones, nowadays people upload a great number of photos to the Internet. Updating photos has become easier, but searching them becomes more difficult. Though the technology of searching images by images has appeared, most people rely on the traditional way to searching an image, which is searching images by typing keywords. For that, we need to add labels for each image, but it cannot be accomplished by human beings due to the great number of unlabeled images. Thus, it is important to use Machine Learning to automatically classify images [1,2] and add correct labels for them.

Multi-Instance Learning is extensively studied in image classification [3]. It uses a kind of data structure called Feature Vector to represent a real image [4], but it is a little bit imprecise, since vectors can only show the pixels of images without the adjacency relations between pixels. Thus, it is natural to consider that Graph may be a better data structure to represent an image [5] because a Graph consists of edges and nodes. Nodes can indicate the texture or color of pixels in an image and the edges can indicate the adjacency relations of nodes. Like the following image in Figure 1a, a Feature Vector can only tell you there are white, blue and green pixels in this image, whereas, after segmenting the image into three Graphs [6] in Figure 1b, Graphs can show the adjacency relations between each pixel. (In the real application, the image will be segmented into more graphs with more nodes.) The latter one represents more real details of an image, which will be more beneficial for the accuracy in the learning part. 


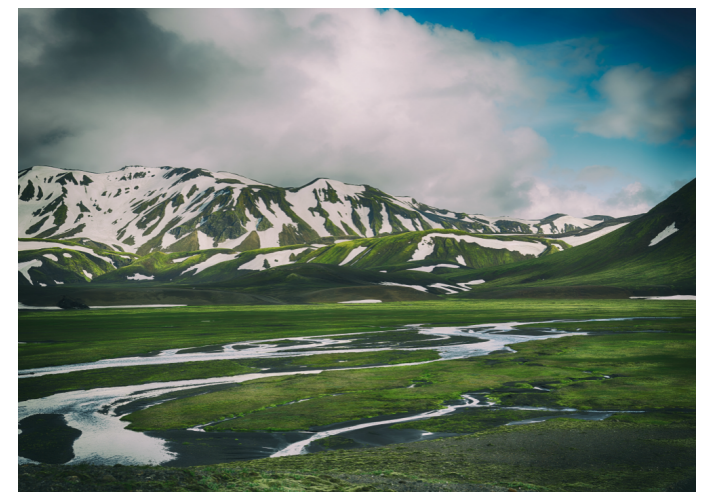

(a) Original image
1:
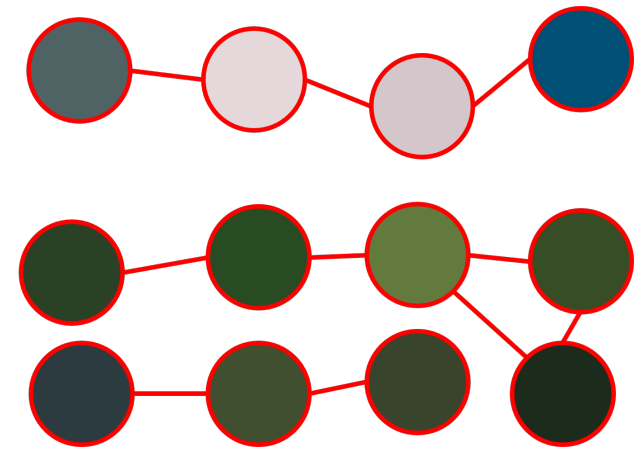

(b) Represented in Graph

Figure 1. An example figure with structure Graph. (a) Original image; (b) Represented in Graph.

Recently, a graph-structure algorithm, namely gMGFL [7-10], was proposed by Jia Wu et al. Briefly, it works in the following steps. Firstly, there are many different images in the training dataset. gMGFL segments each image into multiple graphs, all which are packed into a graph bag. Secondly, the label is only visible for the graph bag and each graph bag will only be marked with one label. For a specific subject, if an image contains it, the label of the graph bag for this image will be positive; on the contrary, it will be negative. Thirdly, to build an appropriate classifier, it needs to mine some informative subgraphs, which can stand for the traits of the subject in the images (or the traits of the subject not in the images), and use these subgraphs as the features for classifying. It is brilliant, but it still has some drawbacks. Two major problems of $g M G F L$ are listed as below.

Firstly, in the algorithm $g M G F L$, each image will only be marked with one label, so it can only deal with one subject. Nevertheless, in the real life, it is impossible that an image just contains one subject. It often includes multiple semantic information [11]. For example, in Figure 2, the image contains three different subjects: sea, boat and sky, so it should be marked with three kinds of positive labels (and maybe also marked with some negative labels, like lion or apple). It cannot mark the image with only one label, like sea. Otherwise, it will cause some problems: if the user types a searching keyword boat or sky, this image will not be shown in the result. Unfortunately, gMGFL can only deal with a one-label problem.

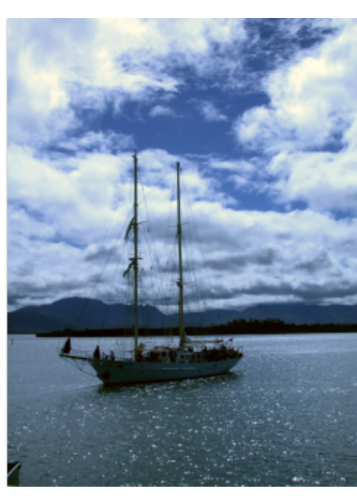

(a) Original image

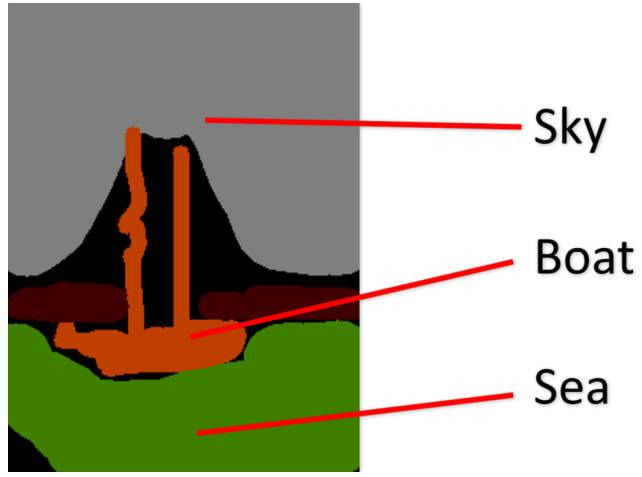

(b) Segmented and labeled

Figure 2. An example figure with Multi-Label. (a) Original image; (b) Segmented and labeled.

Secondly, in the part of mining informative subgraphs, gMGFL considers that if a subgraph is informative, it should be frequent in the dataset. Therefore, $g M G F L$ mines the frequent subgraphs [12] and uses them as the features for classifying, but this is not always accurate. In Figure 3, there are eight graphs and two different classes. The above four are marked with positive labels and belong to the 
positive class; the four below are marked with negative labels and belong to the negative class. If we only consider the frequent-subgraph mining, the subgraph $A-B$ has the frequency of eight because it appears eight times in all graphs. Nevertheless, if we regard it as an informative subgraph (a.k.a. classifying feature), it cannot distinguish the positive class from the negative class, since it is a common feature between two classes. Not only do all positive graphs contain the subgraph $A-B$, but also all negative ones contain it as well. Thus, the subgraph $A-B$ is not appropriate to be an informative feature, but, due to its high frequency, gMGFL considers that it is, which will cause imprecise results.
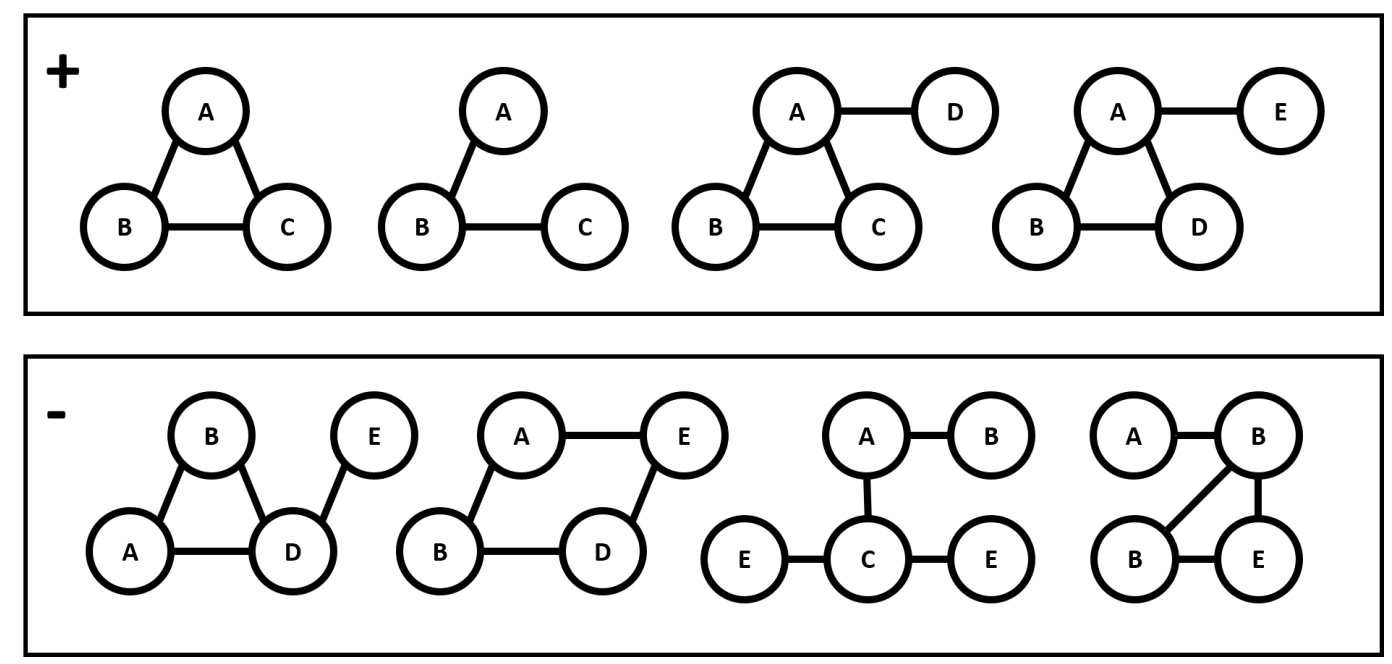

Figure 3. A graph dataset with class label.

To solve these problems, in this paper, we proposed an advanced graph-structure algorithm named Multi-Graph Multi-Label Learning. This algorithm may also be utilized in calculating the similarity of biological sequences, predicting the function of chemical compounds, analyzing structured texts such as HTML (Hypertext Markup Language) and XML (Extensible Markup Language), etc. The following are our major contributions:

1. Our algorithm is based on a multi-graph and it can also solve multi-label (i.e., multiple subjects) problems, which means it can deal with multiple semantic information. To the best of our knowledge, we are the first one to propose such an algorithm.

2. We introduce a novel subgraph-mining technique called Entropy-Based Subgraph Mining. It calculates the information entropy for each graph [13] and uses this criterion to mine the informative subgraphs, which is more suitable than the one based on frequent-subgraph.

3. In the part of building the classifier, we utilize the algorithm MIML-ELM (we will discuss it briefly in Section 2.3). It uses the Extreme Learning Machine rather than Support Vector Machine to build an image classifier, which is more efficient.

The rest part of this paper is organized as the following. Related works are introduced in Section 2. The algorithm description of Multi-Graph Multi-Label Learning is presented in Section 3. The results of our experiments are provided in Section 4. Our conclusions are in Section 5.

\section{Related Work}

The research in this paper is related to some previous works of graph-structure classification, Multi-Instance Multi-Label Learning and MIML-ELM. We will briefly review them respectively in Sections 2.1-2.3. 


\subsection{Graph-Structure Classification}

There are two kinds of algorithms about graph-structure classification: one of them is based on global distance and the other one is based on subgraph feature, and it has been proved that the subgraph-feature approach is better [14]. It converts a set of subgraphs into feature vectors so that most of the current algorithms can be utilized in the graph classification problem. Almost all these kinds of algorithms (such as AGM [15], Gaston [16], gSpan [17,18], gHSIC [19,20]) extract subgraph features by using frequent substructure pattern mining, and the most widespread mining algorithm among them is gSpan.

\subsection{Multi-Instance Multi-Label Learning}

Multi-Instance Multi-Label Learning is a supervised learning algorithm, which represents real-world objects with bags of instances and labels. The most widespread algorithm is MIML-SVM [21]. It degenerates Multi-Instance Multi-Label to Single-Instance Multi-Label by clustering multiple labels to binary classification tasks with Support Vector Machine. Zhou et al. proposed MIML-SVM in [22] and recently Li et al. improved it in [23].

\subsection{MIML-ELM}

The full name of MIML-ELM is Improving Multi-Instance Multi-Label Learning by Extreme Learning Machine [24]. Extreme Learning Machine (ELM) is one of the models in Neural Networks and is extensively utilized in Single Hidden-layer Feed-forward Network. Recently, Li et al. proposed an efficient and effective algorithm named MIML-ELM, which utilized the ELM in solving the Multi-Instance Multi-Label problem. Firstly, this algorithm is more effective in the process of degeneration from Multi-Instance Multi-Label to Single-Instance Multi-Label, since it provides a theoretical guarantee to automatically determine the number of clusters. Secondly, this algorithm is more efficient, since it uses ELM instead of Support Vector Machine to improve the two-phase framework.

\section{The MGML Algorithm}

This section is about the algorithm description of Multi-Graph Multi-Label Learning (MGML). Firstly, we will introduce some relative concepts in Section 3.1. Then, we discuss our proposed approach in Sections 3.2-3.4. Lastly, an illustrative example of MGML is given in Section 3.5.

\subsection{Problem Definition}

The following are some basic definitions about our algorithm.

Definition 1. (Graph Bag): $G$ is a graph denoted as $G=(N, E, L, l) . N$ is a set of nodes; $E$ is a set of edges and $E \subseteq N \times N$; $L$ is a set of labels for nodes and edges; $l$ is the function mapping labels to nodes and edges and $l: N \cup E \rightarrow L$. A graph bag Bag $=\left\{G_{1}, \ldots, G_{j}, \ldots, G_{n}\right\}$ contains $n$ graphs, where $G_{j}$ denotes the $j$-th graph in the bag.

Definition 2. (Subgraph): Given $G=(N, E, L, l)$ and $S u b G_{k}=\left(N^{\prime}, E^{\prime}, L^{\prime}, l^{\prime}\right)$, we say that $S u b G_{k}$ is a subgraph of $G$, if and only if there exists a function $\psi: N^{\prime} \rightarrow N$ s.t. (1) $\forall n \in N^{\prime}, l^{\prime}(n)=l(\psi(n))$; (2) $\forall\left(n_{1}, n_{2}\right) \in E^{\prime},\left(\psi\left(n_{1}\right), \psi\left(n_{2}\right)\right) \in E$, and $l^{\prime}\left(n_{1}, n_{2}\right)=l\left(\psi\left(n_{1}\right), \psi\left(n_{2}\right)\right)$. In addition, we can say that $G$ is a super-graph of $S u b G_{k}$.

Definition 3. (Subgraph Feature Representation for Graph): Let Set ${ }_{S u b G}=\left\{S u b G_{1}, \ldots, S_{u b G_{s}}\right\}$ be a set of subgraphs mined from a graph set $\operatorname{Set}_{G}=\left\{G_{1}, \ldots, G_{t}\right\}$. For each graph $G_{i}(i \in[1, t])$ in $\operatorname{Set}_{G}$, we use a feature vector $X_{i}^{G}=\left[\left(X_{i}^{\text {SubG }}\right)^{G}, \ldots,\left(X_{i}^{\text {SubG } G_{k}}\right)^{G}, \ldots,\left(X_{i}^{\text {SubG } G_{s}}\right)^{G}\right]^{T}$ to represent it. $\left(X_{i}^{\text {SubG }}\right)^{G}=1$ if and only if $S u b G_{k}$ is a subgraph of $G_{i}$. Otherwise, $\left(X_{i}^{S u b G_{k}}\right)^{G}=0$. 


\subsection{Overall Framework of $M G M L$}

The framework of MGML includes two major steps: (1) Mining Informative Subgraphs. Our novel subgraph-mining technique Entropy-based Subgraph Mining will be utilized in this part and it includes the following steps. Firstly, gSpan will be utilized to generate all subgraphs in the dataset. Secondly, entropies of all subgraphs will be calculated and ranked according to our informative-subgraph criterion based on information entropy. We will discuss all details in Section 3.3; (2) Building Classifier. Top-ranked subgraphs will be used as classifying features. Graphs can be represented as instances based on what kinds of classifying features (a.k.a. informative subgraphs) that they contain, so graphs can be represented as multiple instances. Thus, Multi-Graph Multi-Label degenerates to Multi-Instance Multi-Label. After that, we will utilize MIML-ELM, an efficient and effective MIML algorithm to build a classifier. We will discuss all details in Section 3.4.

\subsection{Mining Informative Subgraphs}

In this section, we will discuss the evaluation of informative subgraphs and the algorithm how to mine them.

\subsubsection{Evaluation of Informative Subgraphs}

Let us reconsider the example in Figure 3. Although another subgraph $B-C$ has the frequency of only three, it appears three times in four positive graphs and does not appear in the negative ones at all, so it can stand for the trait of the positive class and is suitable to be regarded as a classifying feature. Generally, if a subgraph appears frequently in one of the classes but hardly appears in the other class, according to the definition of information entropy, this subgraph has low entropy. Thus, the subgraphs that have low entropy are the informative subgraphs that we need. We will give the formal definition of the informative subgraph in the following. Firstly, we will define it in the single-label problem for the ease of understanding and then expand it to a multi-label problem.

Firstly, we give the definition of information entropy for subgraph in the single-label problem. Assume that there is a set of graphs $\operatorname{Set}_{G}=\left\{G_{1}, \ldots, G_{m}\right\}$. Each graph $G_{i}(i \in[1, m])$ is only marked with a single label and the label is either positive or negative. $S t_{S u b G}$ is the complete set of subgraphs mined from $\operatorname{Set}_{G}$, which is denoted as $\operatorname{Set}_{S u b G}=\left\{S u b G_{1}, \ldots, S u b G_{n}\right\}$. For each subgraph $\operatorname{Sub} G_{j}(j \in$ $[1, n])$, the set of super-graphs for it is $\operatorname{Set}_{G}^{j}=\left\{G_{1}^{j}, \ldots, G_{u}^{j}\right\}$. \# $\#_{p o s}$ is the number of positive graphs in $\operatorname{Set}_{G}^{j}$ and $\#_{n e g}$ is the number of negative graphs in $\operatorname{Set}_{G}^{j}\left(\#_{\text {pos }}+\#_{n e g}=u\right)$. Since each graph $G_{k}^{j}(k \in[1, u])$ in $\operatorname{Set}_{G}^{j}$ is the super-graph of $S u b G_{j}$, the possibility of $S u b G_{j}$ appearing in a positive (or negative) class equals the percentage of positive (or negative) graphs in $\operatorname{Set}_{G}^{j}$ (based on Definition 2). Thus, the information entropy of subgraph $S u b G_{j}$ is $E_{j}=-p_{\text {pos }} \log _{2}\left(p_{\text {pos }}\right)-p_{\text {neg }} \log _{2}\left(p_{\text {neg }}\right) . p_{\text {pos }}$ is the possibility of $S u b G_{j}$ appearing in a positive class and $p_{\text {pos }}=\frac{\#_{p o s}}{u} ; p_{\text {neg }}$ is the possibility of $S u b G_{j}$ appearing in a negative class and $p_{\text {neg }}=\frac{\#_{\text {neg }}}{u}$. The information entropy for the set of subgraphs Set $t_{S u b G}$ is $\operatorname{Set}_{E(\operatorname{SubG})}=\left\{E_{1}, \ldots, E_{n}\right\}$.

Furthermore, the following is the definition of information entropy for a subgraph in a multi-label problem. Assume that there is a set of graphs $\operatorname{Set}_{G}=\left\{G_{1}, \ldots, G_{m}\right\}$ and each graph $G_{i}(i \in[1, m])$ is marked with a set of labels $\operatorname{Set}_{L_{i}}=\left\{L_{i, 1}, \ldots, L_{i, t}\right\}$. Set $t_{S u b G}$ is the complete set of subgraphs mined from $S_{e t}{ }_{G}$, which is denoted as $\operatorname{Set}_{S u b G}=\left\{S u b G_{1}, \ldots, S u b G_{n}\right\}$. For each subgraph $S_{u b} G_{j}(j \in[1, n])$, it has a set of information entropy $\operatorname{Set}_{E_{j}}=\left\{E_{j, 1}, \ldots, E_{j, t}\right\}$ ( $t$ different kinds of information entropy for $t$ different labels). We define that, for each subgraph, the information entropy in a multi-label problem is the average entropy of all labels. Thus, for the subgraph $S u b G_{j}$, the information entropy in a multi-label problem is $E_{j}=\operatorname{avg}\left\{E_{j, 1}, \ldots, E_{j, t}\right\}$. In the case of multi-label, the information entropy for the set of subgraphs $\operatorname{Set}_{S u b G}$ is denoted as $\operatorname{Set}_{E(\operatorname{SubG})}=\left\{E_{1}, \ldots, E_{n}\right\}$.

Lastly, $\operatorname{Set}_{E(S u b G)}$ will be ranked increasingly. The top-ranked subgraphs (i.e., the ones with lower entropy) are the informative subgraphs. 


\subsubsection{Entropy-Based Subgraph Mining}

Current algorithms about classifying graph-structure data can be categorized into two groups: one is based on global distance, including graph kernel [25,26], graph embedding [27] and graph transformation [28], which calculates the similarity rate of two graphs; the other one is based on subgraph feature [29], including AGM, Gaston, gSpan and gHSIC, which converts a set of subgraphs to feature vectors. It has been proved that the latter one is better. It converts subgraphs to vectors so that most of the current algorithms can be utilized in the graph-structure classification problem.

To mine informative subgraphs as classifying features, one of the straightforward approaches is to mine the complete set of subgraphs for the graph set and rank these subgraphs with the evaluation function in Section 3.3.1, but this approach will cause a problem: the number of subgraphs grows exponentially when the size of graph set grows, so the enumeration will be tough work. Alternatively, we use a Depth-First-Search (DFS) based on algorithm gSpan to generate all subgraphs, using our evaluation during the process.

The key idea of gSpan is to build a lexicographic order among graphs that need to be mined, and then give each graph a unique label named minimum DFS (Depth-First-Search) code. gSpan uses the strategy of depth-first search to mine the frequent subgraph with the DFS code. Each time it needs to generate a new subgraph, it just recurs the character string (i.e., DFS code), so a subgraph-mining problem can be transformed into a substring-matching problem. Thus, the gSpan performs better than previous similar algorithms.

Generally, gSpan is utilized for mining the frequent subgraphs, but we do not care about the frequency of subgraph. We only use the gSpan to traverse all subgraphs and evaluate the information entropy during the traversal. This is the general idea of Entropy-based Subgraph Mining (ESM). The detailed algorithm of ESM is described in Algorithms 1 and 2.

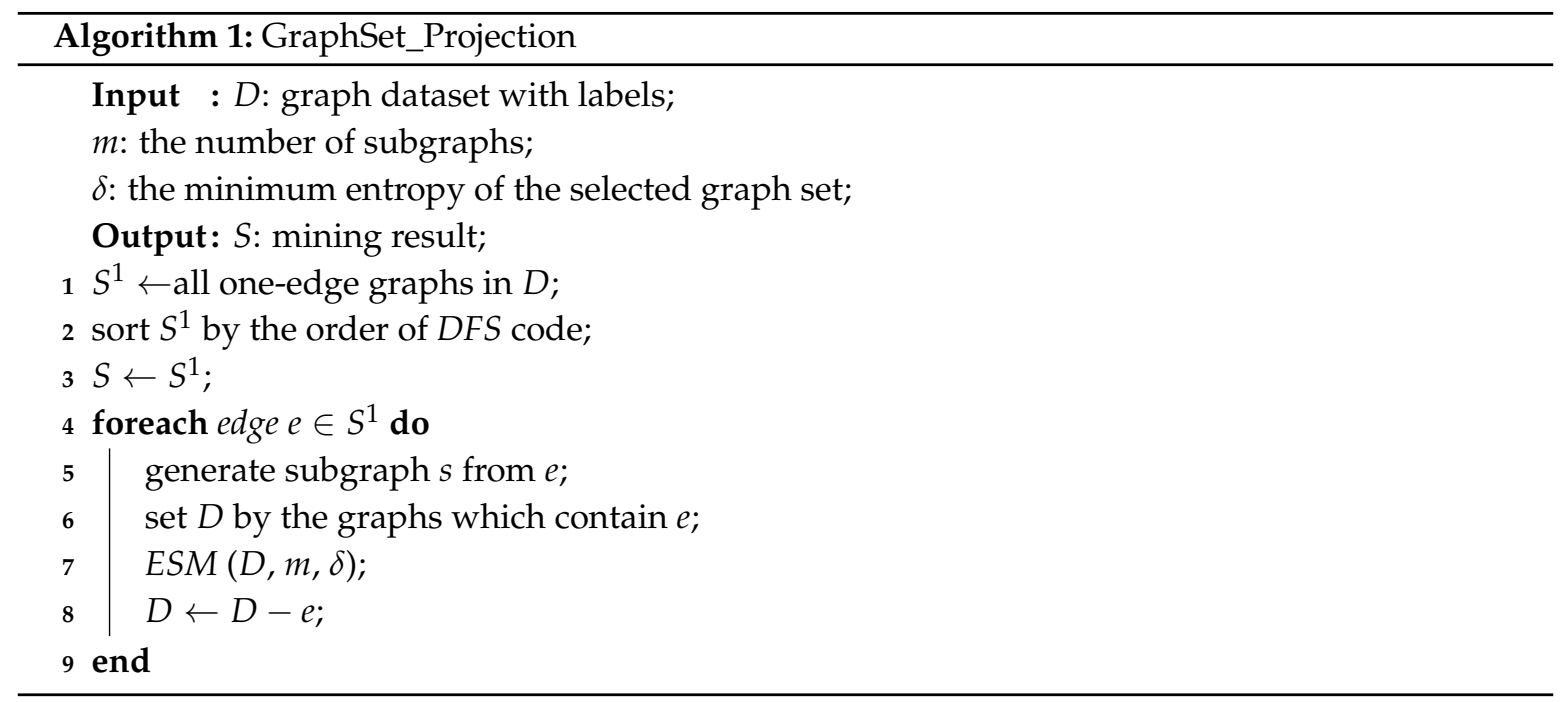

Note that Algorithm 1 invokes Algorithm 2 and Algorithm 2 is a recursion function. Firstly, it generates all subgraphs by traversing the graph from one edge (lines 4-9 in Algorithm 1). The search space is shrunk at the end of each turn (line 8 in Algorithm 1). Algorithm 2 traverses all graphs and generates all their subgraphs. It will stop when the code of the subgraph is not a minimum code (line 1 in Algorithm 2). The information entropy of the subgraph is computed and the result set is built by conducting Lines 4-11 in Algorithm 2. Lines 15-19 in Algorithm 2 grows the subgraph and does this function recursively. 


\subsection{Building Classifier}

Since current algorithms of classifiers cannot be utilized directly in the graph-structure, after mining the informative subgraphs, we need to degenerate Multi-Graph Multi-Label (MGML) to Multi-Instance Multi-Label (MIML) based on Definition 3. The general idea is that, assuming there is a set of graphs $\operatorname{Set}_{G}=\left\{G_{1}, \ldots, G_{m}\right\}$ and a set of informative subgraphs Set $_{\text {InfoSubG }}=$ $\left\{\operatorname{InfoSub}_{1}, \ldots, \operatorname{InfoSub}_{n}\right\}$ mined from $S_{e t}$. For each graph $G_{i}(i \in[1, m])$, it equals a feature vector $V_{i}=\left(x_{1}, \ldots, x_{n}\right)$ (a.k.a. instance) and the dimension of it is $n$ (that equals to the number of informative subgraphs). For each informative subgraph $\operatorname{InfoSubG}_{j}(j \in[1, n])$, if $G_{i}$ is the super-graph of InfoSubG $G_{j}, x_{j}$ in $V_{i}$ has $x_{j}=1$; otherwise, $x_{j}=0$. The labeled MIML set is $D=\left\{\left(B_{i}, Y_{i}\right) \mid i=1, \ldots, N\right\}$, where $N$ is the number of bags in dataset $D$ and $B_{i}=\left\{X_{1}^{i}, \ldots, X_{j}^{i}, \ldots, X_{n_{i}}^{i}\right\}$ is an instance bag with $n_{i}$ instances, $Y_{i} \in\{0,1\}^{M}$ is the label vector of bag $B_{i}$. Now, the MGML problem is degenerated to the MIML problem.

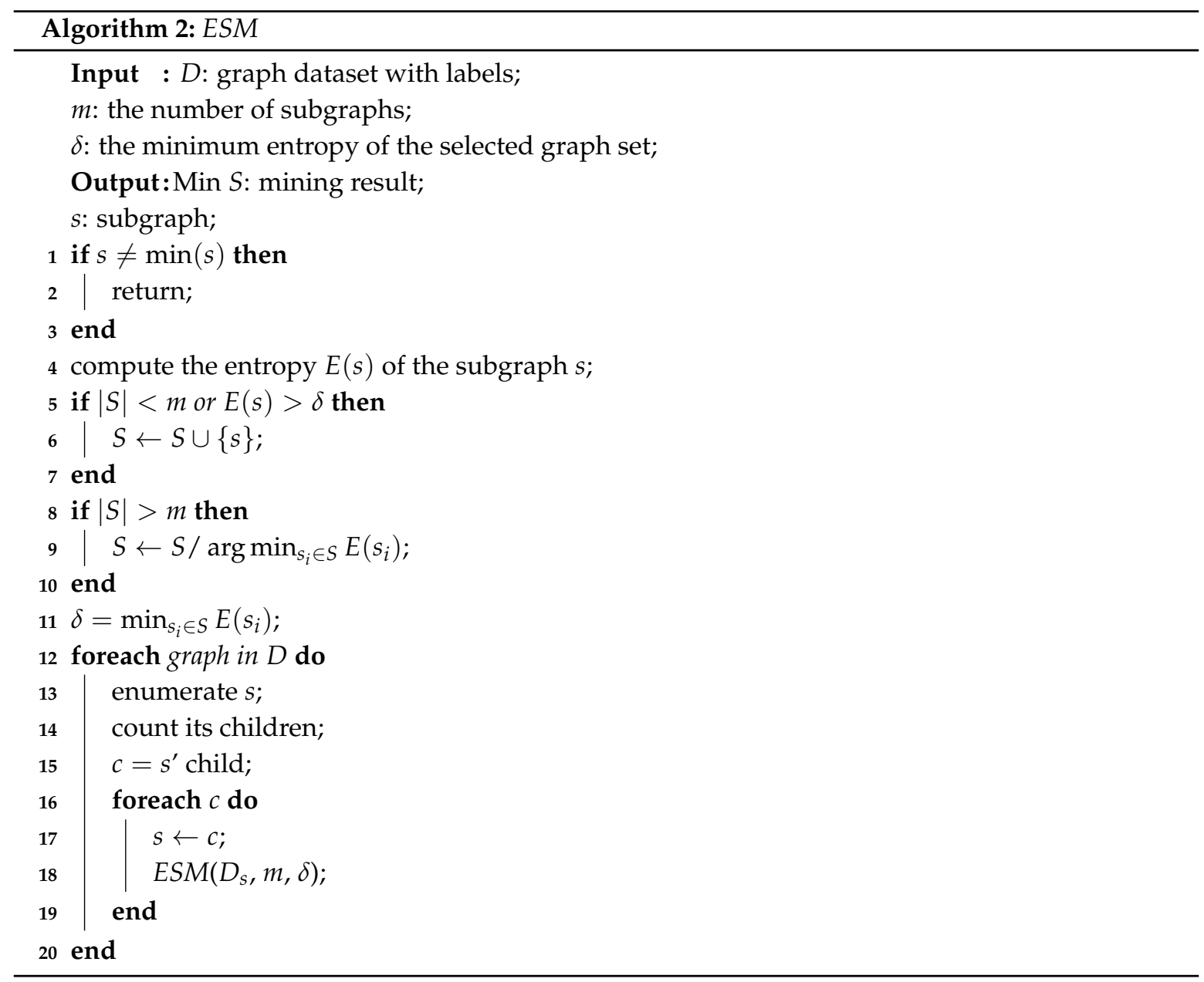

Traditionally, Support Vector Machine (SVM) is utilized to solve the MIML, but it has some drawbacks. First, $S V M$ requires the user to input a great number of parameters. Second, using $S V M$ to build a classifier may cause a high computing cost and the performance depends on the specific parameters that the user defined. Thus, we choose to use the Extreme Learning Machine (ELM) to solve the MIML problem. Firstly, ELM develops a theoretical guarantee to determine the number of clusters by AIC [30]. Secondly, a k-medoids cluster process is performed to transform from Multi-Instance Multi-Label into Single-Instance Multi-Label. Then, the Hausdorff distance [31] is used to measure the similarity between two different multi-instance bags. Based on the Hausdorff distance, 
$k$-medoids cluster divides the dataset into $k$ parts, the medoids of which are $M_{1}, \ldots, M_{k}$. At last, we train the classifier for each label with $k$-dimensional numerical vectors.

\subsection{Example of MGML}

In this section, we will give an explanatory example of $M G M L$. In Figure 4a, there are three images and two labels. In image 1, label Lion is positive $(+)$ and label Tree is positive $(+)$. In image 2, label Lion is positive (+) and label Tree is negative (-). In image 3, label Lion is negative (-) and label Tree is negative (-). The following are the brief steps to build a classifier with MGML.

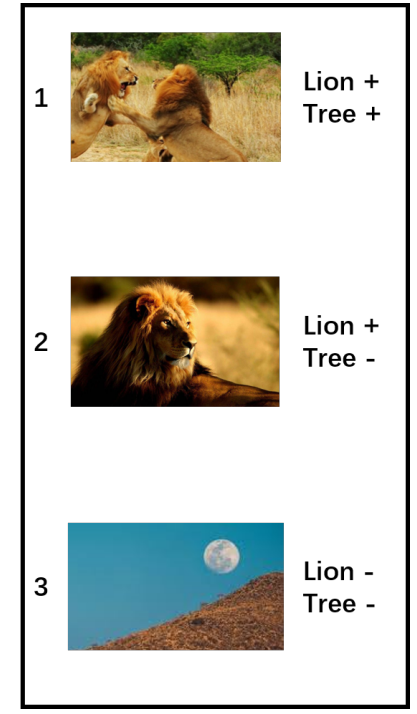

(a) Original images and labels

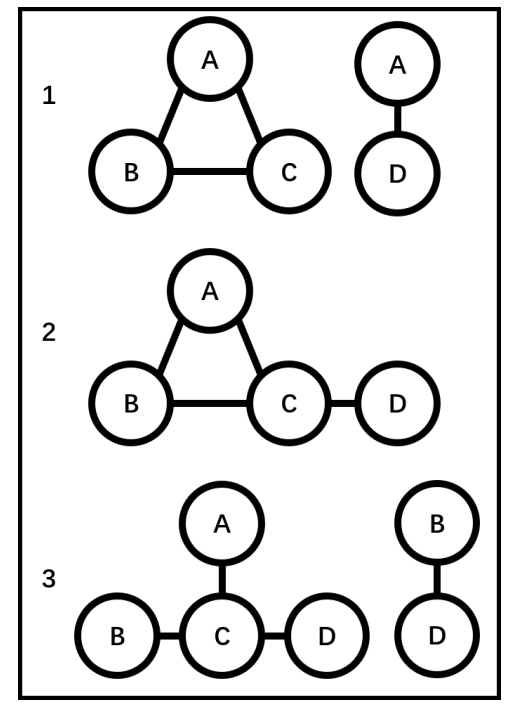

(b) Segmented to multiple graphs

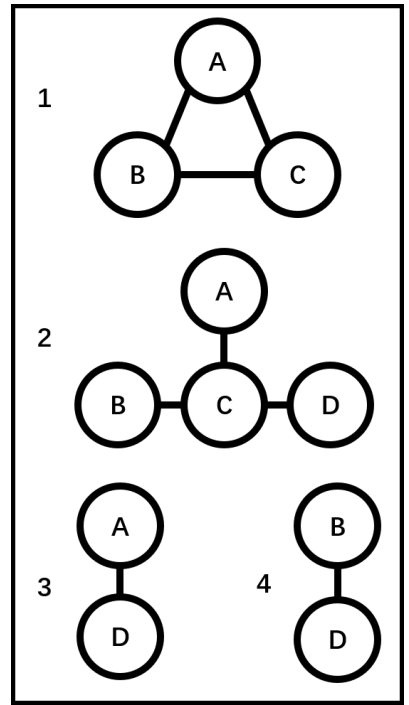

(c) Informative subgraphs

Figure 4. An example of MGML (1). (a) Original images and labels; (b) Segmented to multiple graphs; (c) Informative subgraphs.

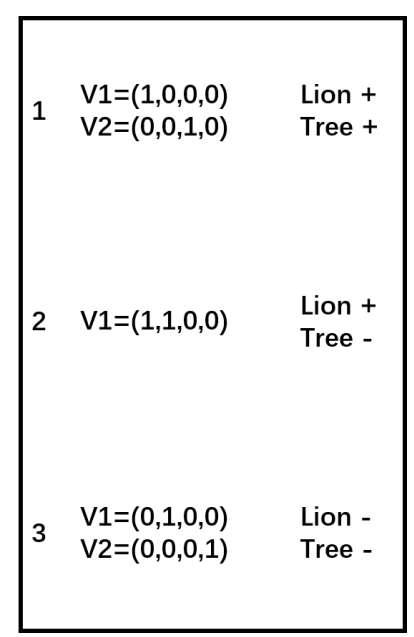

(a) Multiple instances

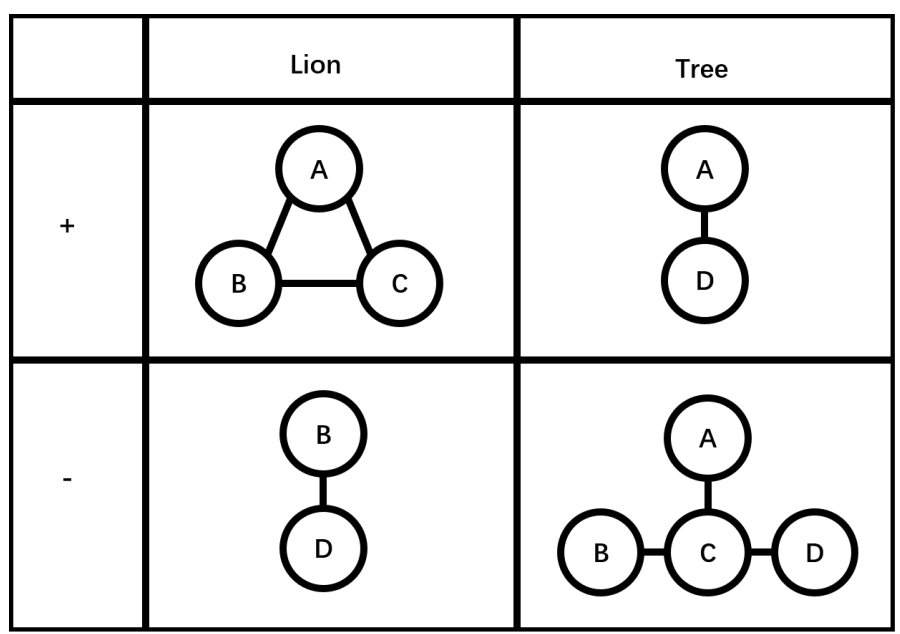

(b) Relation between informative subgraphs and labels

Figure 5. An example of MGML (2). (a) Multiple instances; (b) Relation between informative subgraphs and labels.

Firstly, segment these images into multiple graphs like Figure 4b. Secondly, utilize Entropy-based Subgraph Mining to mine informative subgraphs. The result is in Figure 4c. Thirdly, transform Multi-Graph to Multi-Instance. The result is in Figure 5a. Lastly, utilize MIML-ELM to build a classifier. 
The formula (i.e., relation) between informative subgraphs and labels is in Figure $5 b$. For example, subgraph $A-D$ is the trait when label Tree is negative (-).

Assume that there is a new image without labels in Figure 6a. Segment the image into multiple graphs like Figure $6 \mathrm{~b}$. If we want to add labels for it with our MGML classifier, we just need to see what kinds of classifying features (i.e., the informative subgraphs in the previous step) it contains. It contains $A-D$ and $B-D$, so it should have a negative (-) label Lion and a positive $(+)$ label Tree.

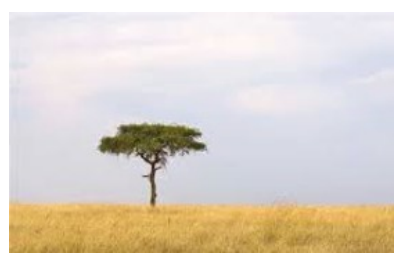

(a) Unlabeled image

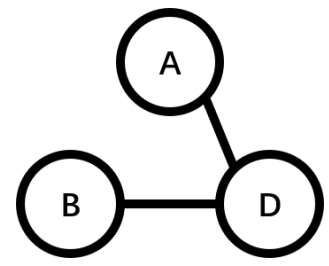

(b) Segmented to multiple graphs

Figure 6. An example of MGML (3). (a) Unlabeled image; (b) Segmented to multiple graphs.

\section{Experimental Section}

The following experiments are performed on a PC running Linux with an Intel dual-core CPU (2.60 GHz) (Shenyang, China) and 16 GB memory.

\subsection{Datasets}

Experiments are performed on three image datasets. These datasets have different sizes, including a small size of dataset named MSRC v2 (Microsoft Research Cambridge) [32], a middle size of dataset named Scenes [22,33] and a large size of dataset named Corel5K [34].

The summary of three datasets is given in Table 1.

Table 1. The summary of datasets.

\begin{tabular}{cccc}
\hline Dataset & \# of Bags & \# of Labels & Labels Per Bag \\
\hline MSRC v2 & 591 & 23 & 2.5 \\
Scenes & 2000 & 5 & 1.2 \\
Corel5K & 5000 & 260 & 3.5 \\
\hline
\end{tabular}

We use two ways to segment the original datasets: the first one is to segment each image into six graph structures and each graph has eight nodes and 12 edges roughly $(6 \times 8 \times 12)$; the second one is to segment each image into six graph structures and each graph has nine nodes and 15 edges roughly $(6 \times 9 \times 15)$. In short, graphs in $6 \times 9 \times 15$ set are more complex to mining than the $6 \times 8 \times 12$ one.

In the following experiments, each dataset will be randomly divided to a training dataset and a testing dataset and the ratio of them is about 2:1. The training dataset will be used to build the classifier and the testing dataset will be used to evaluate its performance. All experiments repeatedly run thirty times, and each time the training dataset and the testing dataset will be divided randomly.

\subsection{Evaluation Criteria}

Assume that there is a test dataset $S=\left\{\left(X_{1}, Y_{1}\right),\left(X_{2}, Y_{2}\right), \ldots,\left(X_{p}, Y_{p}\right)\right\} . h\left(X_{i}\right)$ denotes a set of correct labels of $X_{i} ; h\left(X_{i}, y\right)$ denotes the confidence for $y$ to be a correct label of $X_{i} ; \operatorname{rank}^{h}\left(X_{i}, y\right)$ denotes the rank of $y$ derived from $h\left(X_{i}, y\right)$. The following are four evaluation criteria to measure the performance of our $M G M L$ algorithm.

1. $\quad$ RankingLoss $=\frac{1}{p} \sum_{i=1}^{p} \frac{1}{\left\|Y_{i}|| \mid \bar{Y}_{i}\right\|}\left|\left\{\left(y_{1}, y_{2}\right) \mid h\left(X_{i}, y_{1}\right) \leq h\left(X_{i}, y_{2}\right),\left(y_{1}, y_{2}\right) \in Y_{i} \times \overline{Y_{i}}\right\}\right|$, where $Y_{i}$ denotes the complementary set of $Y_{i}$ in $Y$. It indicates the average fraction of labels that are 
misordered for a specific object. The smaller the value of RankingLoss is, the better the performance reaches. When it equals to 0 , the performance reaches perfect.

2. OneError $=\frac{1}{p} \sum_{i=1}^{p}\left[\arg \max _{y \in Y} h\left(X_{i}, y\right) \notin Y_{i}\right]$. It indicates the average time that the top labels in the rank are not the correct ones for a specific object. The smaller the value of OneError is, the better the performance reached. When it equals 0 , the performance can reach perfection.

3. Coverage $=\frac{1}{p} \max _{y \in Y} \operatorname{rank}^{h}\left(X_{i}, y\right)-1$. It indicates the average number of labels in the rank that need to be included to cover all the correct labels for a specific object. The smaller the value of Coverage is, the better the performance.

4. Average Precision $\operatorname{avgprec}_{S}(h)=\frac{1}{p} \sum_{i=1}^{p} \frac{1}{\left|Y_{i}\right|} \sum_{y \in Y_{i}} \frac{\left|\left\{y^{\prime} \mid \operatorname{rank}^{h}\left(X_{i}, y^{\prime}\right) \leq \operatorname{rank}^{h}\left(X_{i}, y^{\prime}\right) \leq \operatorname{rank}^{h}\left(X_{i}, y\right), y^{\prime} \in Y_{i}\right\}\right|}{\operatorname{rank}^{h}\left(X_{i}, y\right)}$. It indicates the the average fraction of correct labels in all labels $Y_{i}$. The larger the value of Average Precision is, the better the performance. When it equals 1 , the performance can reach perfection.

\subsection{Effectiveness}

In this section, we will use (1), (2) and (3) to mark different algorithms for the differentiation, and we will call our MGML algorithm (1) MGML-ELM, which means that "the MGML algorithm using MIML-ELM".

Currently, there are no other methods of $M G M L$ learning that can be compared to our algorithm. Thus, we use the (3) MIML-SVM, one of the state-of-the-art algorithms for MIML learning, as the competitor. In addition, we use the (2) MGML-SVM as the baseline algorithm for competitions. The (2) $M G M L-S V M$ algorithm is generally the same as the (1) MGML-ELM. It also needs to degenerate the $M G M L$ problem into the MIML problem, but then it uses the SVM instead of ELM in the next step.

The parameter of (1) MGML-ELM is the number of hidden layer $(h n)$, which is respectively set to $50,100,150,200$; the parameter of (2) MGML-SVM and (3) MIML-SVM is the penalty factor of Gaussian kernel (Cost), which is respectively set to $1,2,3,4,5$. The final results on average are in Tables 2-4. The bold one means the best performance for every criterion. The $\downarrow$ indicates the smaller the better, while the $\uparrow$ indicates the larger the better.

Table 2. MSRC v2 Dataset.

\begin{tabular}{lccccc}
\hline \multirow{2}{*}{ MSRC v2 } & & \multicolumn{4}{c}{ Evaluation Criterion } \\
\cline { 3 - 6 } & & RankingLoss $\downarrow$ & OneError $\downarrow$ & Coverage $\downarrow$ & Average Precision $\uparrow$ \\
\hline \multirow{3}{*}{ (1) MGML-ELM } & $\mathrm{hn}=50$ & 0.070079 & 0.183039 & 3.92824 & 0.809013 \\
& $\mathrm{hn}=\mathbf{1 0 0}$ & $\mathbf{0 . 0 7 1 3 6 7}$ & $\mathbf{0 . 1 7 2 5 8 9}$ & $\mathbf{3 . 9 2 8 9 3 4}$ & $\mathbf{0 . 8 2 0 3 8 8}$ \\
& $\mathrm{hn}=150$ & 0.075182 & 0.19797 & 3.989848 & 0.804771 \\
& $\mathrm{hn}=200$ & 0.07181 & 0.187817 & 3.86802 & 0.808192 \\
\hline & Cost $=1$ & 0.154664 & 0.19797 & 7.35533 & 0.754622 \\
& (2) MGML-SVM $=2$ & 0.171189 & 0.229066 & 7.122844 & 0.761665 \\
& Cost $=3$ & 0.183357 & 0.233503 & 7.634518 & 0.735131 \\
& Cost $=4$ & 0.140284 & 0.219557 & 7.628352 & 0.735253 \\
& Cost $=\mathbf{5}$ & $\mathbf{0 . 1 3 7 3 6 1}$ & $\mathbf{0 . 1 8 7 8 1 7}$ & $\mathbf{6 . 1 8 7 8 1 7}$ & $\mathbf{0 . 7 6 2 1 1 5}$ \\
\hline & Cost $=1$ & 0.105581 & 0.295073 & 5.267476 & 0.710714 \\
& Cost $=2$ & 0.104209 & 0.292204 & 5.218223 & 0.715079 \\
(3) MIML-SVM & Cost $=3$ & 0.100998 & 0.253995 & 5.044584 & 0.721987 \\
& Cost $=4$ & 0.097587 & 0.247775 & 4.890471 & 0.73787 \\
& Cost $=\mathbf{5}$ & $\mathbf{0 . 0 9 5 5}$ & $\mathbf{0 . 2 4 0 6 8 2}$ & $\mathbf{4 . 8 8 0 8 9 7}$ & $\mathbf{0 . 7 4 5 3 2 2}$ \\
\hline
\end{tabular}

For ease of reading, we use (1), (2) and (3) in this paragraph to respectively indicate (1) $M G M L-E L M$ (2) MGML-SVM and (3) MIML-SVM. As seen from the results in Table 2, in the dataset MSRC v2, our algorithm (1) performs best when the $h n=100$ and the precision reaches $82 \%$, RankingLoss reaches 0.07 , OneError reaches 0.17 and Coverage reaches 3.93, while the precision of (2) reaches $76 \%$ at best when Cost $=5$, RankingLoss reaches 0.14, OneError reaches 0.19 and Coverage reaches 6.19, and (3) 
reaches $75 \%$ at best when Cost $=5$, RankingLoss reaches 0.10 , OneError reaches 0.24 and Coverage reaches 4.88 .

Table 3. Scenes Dataset.

\begin{tabular}{|c|c|c|c|c|c|}
\hline \multirow{2}{*}{ Scene } & & \multicolumn{4}{|c|}{ Evaluation Criterion } \\
\hline & & RankingLoss $\downarrow$ & OneError $\downarrow$ & Coverage $\downarrow$ & Average Precision $\uparrow$ \\
\hline \multirow{4}{*}{ (1) $M G M L-E L M$} & $\mathrm{hn}=50$ & 0.16927 & 0.318 & 1.771 & 0.798919 \\
\hline & $\mathrm{hn}=100$ & 0.172833 & 0.33 & 1.81 & 0.798367 \\
\hline & $\mathrm{hn}=150$ & 0.165 & 0.304 & 1.78 & 0.811867 \\
\hline & $\mathrm{hn}=200$ & 0.160667 & 0.312 & 1.762 & 0.8102 \\
\hline \multirow{5}{*}{ (2) $M G M L-S V M$} & Cost $=1$ & 0.299667 & 0.806 & 1.324 & 0.555683 \\
\hline & Cost $=2$ & 0.298835 & 0.434 & 1.324 & 0.694689 \\
\hline & Cost $=3$ & 0.2935 & 0.34 & 1.284 & 0.7401 \\
\hline & Cost $=4$ & 0.252933 & 0.36 & 1.312 & 0.630968 \\
\hline & Cost $=5$ & 0.237167 & 0.458 & 1.062 & 0.71515 \\
\hline \multirow{5}{*}{ (3) $M I M L-S V M$} & Cost $=1$ & 0.910205 & 0.950815 & 3.810687 & 0.242251 \\
\hline & Cost $=2$ & 0.91073 & 0.95178 & 3.844675 & 0.242479 \\
\hline & Cost $=3$ & 0.91348 & 0.956172 & 3.864988 & 0.245989 \\
\hline & Cost $=4$ & 0.914378 & 0.957471 & 3.86676 & 0.246726 \\
\hline & Cost $=5$ & 0.917939 & 0.958322 & 3.867907 & 0.249013 \\
\hline
\end{tabular}

As seen from the results in Table 3, in the dataset Scenes, our (1) reaches $81 \%$ at best when the $h n=150$, RankingLoss reaches 0.17, OneError reaches 0.30 and Coverage reaches 1.78, while (2) reaches $74 \%$ at best when Cost $=3$, RankingLoss reaches 0.29, OneError reaches 0.34 and Coverage reaches 1.28 , and (3) reaches $25 \%$ at best when Cost $=5$, RankingLoss reaches 0.92, OneError reaches 0.96 and Coverage reaches 3.87 .

Table 4. Corel5K Dataset.

\begin{tabular}{|c|c|c|c|c|c|}
\hline \multirow{2}{*}{ Corel5K } & & \multicolumn{4}{|c|}{ Evaluation Criterion } \\
\hline & & RankingLoss $\downarrow$ & OneError $\downarrow$ & Coverage $\downarrow$ & Average Precision $\uparrow$ \\
\hline \multirow{4}{*}{ (1) $M G M L-E L M$} & $\mathrm{hn}=50$ & 0.202493 & 0.750168 & 113.8968 & 0.224968 \\
\hline & $\mathrm{hn}=100$ & 0.197103 & 0.743487 & 113.354709 & 0.224146 \\
\hline & $\mathrm{hn}=150$ & 0.21584 & 0.755511 & 120.549098 & 0.219783 \\
\hline & $\mathrm{hn}=200$ & 0.205424 & 0.751503 & 119.306613 & 0.225752 \\
\hline \multirow{5}{*}{ (2) $M G M L-S V M$} & Cost $=1$ & 0.30124 & 0.857229 & 139.0005 & 0.120073 \\
\hline & Cost $=2$ & 0.301264 & 0.86724 & 140.9756 & 0.121792 \\
\hline & Cost $=3$ & 0.301906 & 0.868129 & 141.8067 & 0.122804 \\
\hline & Cost $=4$ & 0.304838 & 0.870358 & 143.3142 & 0.123633 \\
\hline & Cost $=5$ & 0.307872 & 0.880693 & 144.8687 & 0.128766 \\
\hline \multirow{5}{*}{ (3) $M I M L-S V M$} & Cost $=1$ & 0.191867 & 0.768118 & 110.4207 & 0.217195 \\
\hline & Cost $=2$ & 0.191899 & 0.768204 & 110.4322 & 0.217209 \\
\hline & Cost $=3$ & 0.191922 & 0.768299 & 110.4657 & 0.217219 \\
\hline & Cost $=4$ & 0.191978 & 0.768416 & 110.4719 & 0.217285 \\
\hline & $\operatorname{Cos} t=5$ & 0.191997 & 0.768899 & 110.5187 & 0.217299 \\
\hline
\end{tabular}

As seen from the results in Table 4,in the dataset Corel5K, our (1) reaches $23 \%$ at best when the $h n=200$, RankingLoss reaches 0.21 , OneError reaches 0.75 and Coverage reaches 119.31, while (2) reaches $13 \%$ at best when Cost $=5$, RankingLoss reaches 0.31, OneError reaches 0.88 and Coverage reaches 144.87, and (3) reaches $22 \%$ at best when Cost $=5$, RankingLoss reaches 0.19 , OneError reaches 0.77 and Coverage reaches 110.52. Thus, we can say that our (1) MGML-ELM achieves the best performance in all cases. 


\subsection{Efficiency}

In this section, we test the efficiency of our MGML algorithm. For either MGML or MIML, as long as the input numbers of features for ELM and SVM are the same, the runtime for both of these two algorithms will be equal. Therefore, the only way to test the efficiency is focusing on the part of mining the classifying features. Our MGML uses an innovate technique Entropy-based Subgraph Mining (ESM) to mine the informative subgraphs as the features, while the $g M G F L$ of Jia Wu mines the frequent subgraphs as the features, which is based on the boosting gSpan named gboost [35]. We respectively compared the time of our ESM for mining subgraphs with gboost in three datasets: MSRC v2, Scenes and Corel5K.

We implement ESM and gboost in two kinds of segmented datasets $(6 \times 9 \times 15$ and $6 \times 8 \times 12)$ and the minimum frequency is set to $5 \%, 10 \%, 15 \%$, and $20 \%$. In $E S M$, if the frequency of a graph is lower than the minimum frequency we set, we will not calculate the entropy for this graph; while, in gboost, if the frequency of a graph is lower than the minimum frequency, we will not continue to mine the subgraphs for this graph. In short, the lower the minimum frequency is, the more graphs the algorithm needs to mine. The final results in average are in Figure $7 \mathrm{a}-\mathrm{c}$. The time results are logarithmic.

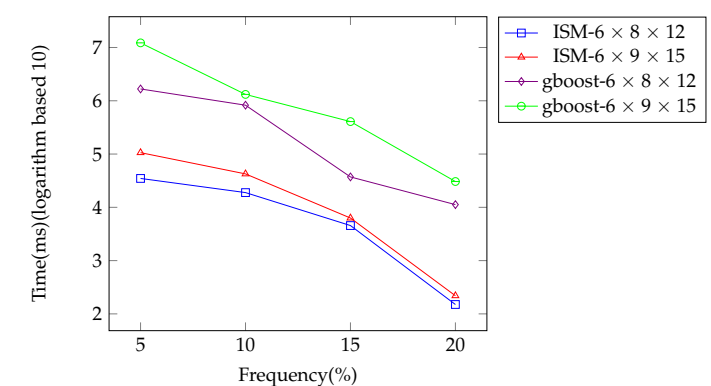

(a) MSRC v2 Dataset

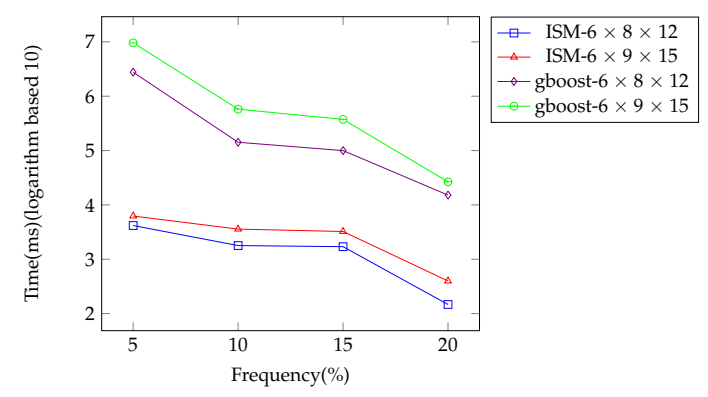

(b) Scenes Dataset

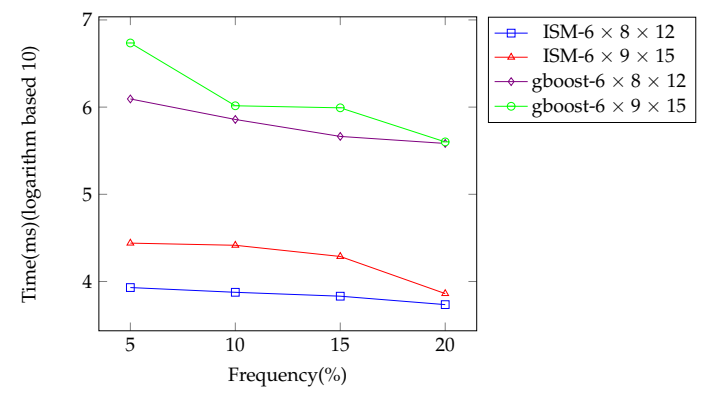

(c) Corel5K Dataset

Figure 7. Results of efficiency experiments. (a) MSRC v2 Dataset; (b) Scenes Dataset; (c) Corel5K Dataset.

As seen from the results in Figure 7a-c, gboost takes hours to mine the huge datasets, but ESM takes only several minutes to generate results. For example, in the dataset MSRC v2 $(6 \times 9 \times 15)$, when the minimum frequency is set to $5 \%, E S M$ takes two minutes to mine the results while gboost takes $4 \mathrm{~h}$ to do that; in the dataset Scenes $(6 \times 9 \times 15)$, when the minimum frequency is set to $10 \%$, ESM takes $4 \mathrm{~s}$ to mine the results while gboost takes $10 \mathrm{~min}$ to do that; in the dataset Corel5K $(6 \times 9 \times 15)$, when the minimum frequency is set to $15 \%$, ESM takes $19 \mathrm{~s}$ to mine the results while gboost takes $16 \mathrm{~min}$ to do that. These figures show that ESM achieves better performance by 100-1000 times in comparison with gboost.

\section{Conclusions}

In this paper, we have shown how the Multi-Graph Multi-Label Learning (MGML) works. The $M G M L$ uses the more precise structures, multiple graphs, instead of instances to represent an 
image, which can improve the accuracy of classification dramatically in the latter step. In addition, it uses multiple labels as the output to eliminate the ambiguity of description. Furthermore, we use our technique Entropy-based Subgraph Mining to mine the informative subgraphs, rather than simply regard frequent subgraphs as informative subgraphs. Then, we show how to degenerate MGML to MIML. At last, we use the MIML-ELM as the base classifier. Extensive experimental results prove that $M G M L$ achieves a good performance in three image datasets with different sizes. What we are interested in for the future steps is to improve the performance in the dataset that has sparse labels by using other algorithms as the classifier.

Acknowledgments: This work was supported by the National Natural Science Foundation Program of China (61772124 and 61702381), the State Key Program of National Natural Science of China (61332014), the National Training Program of Innovation and Entrepreneurship for Undergraduates (201710145082), the Fundamental Research Funds for the Central Universities under Grant 150402002, and the Peak Discipline Construction of Computer Science and Technology under Grant 02190021821001.

Author Contributions: Zixuan Zhu and Yuhai Zhao conceived and designed the experiments; Zixuan Zhu and Yuhai Zhao wrote and revised the paper; Yuhai Zhao made substantial contributions to the conception of the work; Zixuan Zhu acquired and analyzed the data; and Zixuan Zhu performed the experiments.

Conflicts of Interest: The authors declare no conflict of interest.

\section{References}

1. Wang, W.; Vong, C.; Yang, Y.; Wong, P. Encrypted image classification based on multilayer extreme learning machine. Multidimens. Syst. Signal Process. 2017, 28, 851-865.

2. Yan, K.; Li, Z.; Zhang, C. A New multi-instance multi-label learning approach for image and text classification. Multimed. Tools Appl. 2016, 75, 7875-7890.

3. Tang, P.; Wang, X.; Feng, B.; Liu, W. Learning Multi-Instance Deep Discriminative Patterns for Image Classification. IEEE Trans. Image Process. 2017, 26, 3385-3396.

4. Chaiyakhan, K.; Kerdprasop, N.; Kerdprasop, K. Feature Selection Techniques for Breast Cancer Image Classification with Support Vector Machine. Lect. Notes Eng. Comput. Sci. 2016, 2221, 327-332.

5. Bashier, H.; Hoe, L.; Hui, L.; Azli, M.; Han, P.; Kwee, W.; Sayeed, M. Texture classification via extended local graph structure. Optik Int. J. Light Electron Opt. 2016, 127, 638-643.

6. Wang, W.; Li, Z.; Yue, J.; Li, D. Image segmentation incorporating double-mask via graph cuts. Comput. Electr. Eng. 2016, 54, 246-254.

7. Wu, J.; Pan, S.; Zhu, X.; Zhang, C.; Wu, X. Positive and Unlabeled Multi-Graph Learning. IEEE Trans. Cybern. 2017, 47, 818-829.

8. Wu, J.; Hong, J.; Pan, S.; Zhu, X.; Cai, Z.; Zhang, C. Multi-graph-view subgraph mining for graph classification. Knowl. Inf. Syst. 2016, 48, 29-54.

9. Wu, J.; Zhu, X.; Zhang, C.; Yu, P.S. Bag Constrained Structure Pattern Mining for Multi-Graph Classification. IEEE Trans. Knowl. Data Eng. 2014, 26, 2382-2396.

10. Wu, J.; Zhu, X.; Zhang, C.; Cai, Z. Multi-instance Multi-graph Dual Embedding Learning. In Proceedings of the 2013 IEEE 13th International Conference on Data Mining, Dallas, TX, USA, 7-10 December 2013; pp. 827-836.

11. Wei, Y.; Xia, W.; Lin, M.; Huang, J.; Ni, B.; Dong, J.; Zhao, Y.; Yan, S. HCP: A Flexible CNN Framework for Multi-Label Image Classification. IEEE Trans. Pattern Anal. Mach. Intell. 2016, 38, 1901-1907.

12. Nair, J.; Thomas, S.; Thampi, S.; El-Alfy, E. Improvised Apriori with frequent subgraph tree for extracting frequent subgraphs. J. Intell. Fuzzy Syst. 2017, 32, 3209-3219.

13. Bai, L.; Hancock, E. Fast depth-based subgraph kernels for unattributed graphs. Pattern Recognit. 2016, 50, 233-245.

14. Ketkar, N.; Holder, L.; Cook, D. Empirical Comparison of Graph Classification Algorithms. In Proceedings of the IEEE Symposium Computational Intelligence and Data Mining (CIDM), Nashville, TN, USA, 30 March-2 April 2009; pp. 259-266.

15. Inokuchi, A.; Washio, T.; Motoda, H. An Apriori-Based Algorithm for Mining Frequent Substructures from Graph Data. In Proceedings of the Fourth European Conference on Principles of Data Mining and Knowledge Discovery (PKDD), Lyon, France, 13-16 September 2000; pp. 13-23. 
16. Nijssen, S.; Kok, J. A Quickstart in Frequent Structure Mining can Make a Difference. In Proceedings of the 10th ACM SIGKDD International Conference on Knowledge Discovery and Data Mining (KDD), Seattle, WA, USA, 22-25 August 2004; pp. 647-652.

17. Saigo, H.; Nowozin, S.; Kadowaki, T.; Kudo, T.; Tsuda, K. gBoost: A Mathematical Programming Approach to Graph Classification and Regression. Mach. Learn. 2009, 75, 69-89.

18. Yan, X.; Han, J. gSpan: Graph-Based Substructure Pattern Mining. In Proceedings of the IEEE International Conference on Data Mining (ICDM), Maebashi, Japan, 9-12 December 2002; pp. 721-724.

19. Zhao, Y.; Kong, X.; Yu, P.S. Positive and Unlabeled Learning for Graph Classification. In Proceedings of the IEEE 11th International Conference on Data Mining (ICDM), Vancouver, BC, Canada, 11-14 December 2011; pp. 962-971.

20. Kong, X.; Yu, P. Multi-Label Feature Selection for Graph Classification. In Proceedings of the 10th International Conference on Data Mining (ICDM), Sydney, Australia, 13-17 December 2010; pp. 274-283.

21. Wu, J.; Huang, S.; Zhou, Z. Genome-wide protein function prediction through multi-instance multi-label learning. IEEE/ACM Trans. Comput. Biol. Bioinform. 2014, 11, 891-902.

22. Zhou, Z.; Zhang, M. Multi-instance multi-label learning with application to scene classification. In Advances in Neural Information Processing Systems; The MIT Press: Cambridge, MA, USA, 2007; pp. 1609-1616.

23. Li, Y.; Ji, S.; Kumar, S.; Ye, J.; Zhou, Z. Drosophila gene expression pattern annotation through multi-instance multi-label learning. IEEE/ACM Trans. Comput. Biol. Bioinform. 2012, 9, 98-112.

24. Yin, Y.; Zhao, Y.; Li, C.; Zhang, B. Improving Multi-Instance Multi-Label Learning by Extreme Learning Machine. Appl. Sci. 2016, 6, 160.

25. Martino, G.; Navarin, N.; Sperduti, A. Graph Kernels exploiting Weisfeiler-Lehman Graph Isomorphism Test Extensions. Neural Inf. Process. 2014, 8835, 93-100.

26. Seeland, M.; Kramer, A.K.S. A Structural Cluster Kernel for Learning on Graphs. In Proceedings of the 18th ACM SIGKDD International Conference on Knowledge Discovery and Data Mining (KDD), Beijing, China, 12-16 August 2012; pp. 516-524.

27. Riesen, K.; Bunke, H. Graph Classification by Means of Lipschitz Embedding. IEEE Trans. Syst. Man Cybern. Part B Cybern. 2009, 39, 1472-1483.

28. Hidaka, S.; Tisi, M.; Cabot, J.; Hu, Z. Feature-based classification of bidirectional transformation approaches. Softw. Syst. Model. 2016, 15, 907-928.

29. Deshpande, M.; Kuramochi, M.; Wale, N.; Karypis, G. Frequent Substructure-Based Approaches for Classifying Chemical Compounds. IEEE Trans. Knowl. Data Eng. 2005, 17, 1036-1050.

30. Maruping, L.; Venkatesh, V.; Brown, S. Going beyond intention: Integrating behavioral expectation into the unified theory of acceptance and use of technology. J. Assoc. Inf. Sci. Technol. 2017, 68, 623-637.

31. Balankin, A.; Mena, B.; Cruz, M. Topological Hausdorff dimension and geodesic metric of critical percolation cluster in two dimensions. Phys. Lett. A 2017, 381, 2665-2672.

32. Winn, J.; Criminisi, A.; Minka, T. Object categorization by learned universal visual dictionary. In Proceedings of the Tenth IEEE International Conference on Computer Vision, Beijing, China, 17-21 October 2005; Volume 2; pp. 1800-1807.

33. Zhou, Z.; Zhang, M.; Huang, S.; Li, Y. Multi-instance multilabel learning. Artif. Intell. 2012, 176, $2291-2320$.

34. Duygulu, P.; Barnard, K.; Freitas, J.; Forsyth, D. Object recognition as machine translation: Learning a lexicon for a fixed image vocabulary. In Proceedings of the 7th European Conference on Computer Vision, Copenhagen, Denmark, 28-31 May 2002; pp. 97-112.

35. Nowozin, S.; Tsuda, K.; Uno, T.; Kudo, T; Bakir, G. Weighted Substructure Mining for Image Analysis. In Proceedings of the IEEE Conference on Computer Vision and Pattern Recognition, Minneapolis, MN, USA, 17-22 June 2007; pp. 1-8.

(C) 2018 by the authors. Licensee MDPI, Basel, Switzerland. This article is an open access article distributed under the terms and conditions of the Creative Commons Attribution (CC BY) license (http://creativecommons.org/licenses/by/4.0/). 